\title{
Spectral energy contributions of quasi-periodic oscillations ( $2-35$ days) to the variability of the $f_{0} \mathrm{~F} 2$
}

\author{
E. M. Apostolov ${ }^{1}$, D. Altadill ${ }^{2}$ and R. Hanbaba ${ }^{3}$ \\ ${ }^{1}$ Geophysical Institute, Bulgarian Academy of Sciences, Acad. G. Bonchev Str., bl. 3, 1113 Sofia, Bulgaria \\ ${ }^{2}$ Observatori de l'Ebre, CSIC, URL, Horta Alta 38, 43520-Roquetes (Tarragona), Spain \\ ${ }^{3}$ France Telecom/CNET/Centre Lannion B, Technopole Anticipa 2 Avenue Pierre Marzin, 22307 Lannion Cedex, France
}

Received: 17 February 1997 / Revised: 23 July 1997 / Accepted: 29 July 1997

\begin{abstract}
The relative contributions of quasi-periodic oscillations from 2 to 35 days to the variability of $f_{\mathrm{o}} \mathrm{F} 2$ at middle northern latitudes between $42^{\circ} \mathrm{N}$ and $60^{\circ} \mathrm{N}$ are investigated. The $f_{\mathrm{o}} \mathrm{F} 2$ hourly data for the whole solar cycle 21 (1976-1986) for four European ionospheric stations Rome $\left(41.9^{\circ} \mathrm{N}, 12.5^{\circ} \mathrm{E}\right)$, Poitiers $\left(46.5^{\circ} \mathrm{N}\right.$, $\left.0.3^{\circ} \mathrm{E}\right)$, Kaliningrad $\left(54.7^{\circ} \mathrm{N}, 20.6^{\circ} \mathrm{E}\right)$ and Uppsala $\left(59.8^{\circ} \mathrm{N}, 17.6^{\circ} \mathrm{E}\right)$ are used for analysis. The relative contributions of different periodic bands due to planetary wave activity and solar flux variations are evaluated by integrated percent contributions of spectral energy for these bands. The observations suggest that a clearly expressed seasonal variation of percent contributions exists with maximum at summer solstice and minimum at winter solstice for all periodic bands. The contributions for summer increase when the latitude increases. The contributions are modulated by the solar cycle and simultaneously influenced by the long-term geomagnetic activity variations. The greater percentage of spectral energy between 2 to 35 days is contributed by the periodic bands related to the middle atmosphere planetary wave activity.
\end{abstract}

Key words Ionosphere $\cdot$ Ionosphere-atmosphere interactions $\cdot$ Mid-latitude ionosphere $\cdot$ Plasma waves and instabilities

\section{Introduction}

The existence and persistence of quasi-periodic oscillations with periods from 2 to 35 days, that play an important role in the variability of the ionospheric parameters, are found in many investigations. Some of

Correspondence to: D. Altadill these oscillations have characteristics of planetary waves, which have been observed in the stratosphere and mesosphere and theoretically predicted as free Rossby modes (Salby, 1984). Westward travelling quasi-2-day oscillations are detected in the electron density variations of the lower and upper ionosphere (Pancheva,1988; Pancheva et al., 1994) and a connection with the analogous variations in the mesospheric and lower thermospheric wind and temperature structures has been shown. Chen (1992) reports a quasi-2-day modulation of the equatorial F-region anomaly. Fiveday oscillations in the lower ionosphere (Fraser, 1977) and in the upper ionosphere (Apostolov et al., 1994) have been observed. Forbes and Leveroni (1992) reported a quasi-16-day oscillation in the $E$ and $F$ regions of the equatorial ionosphere.

Important periodicities, close to $2.5,3,6,7,9,10-11$ and 16-days, have been found in the equatorial electrojet variations during 1979 (Parish et al., 1994). The authors suggest that these oscillations have characteristics of planetary waves which can frequently propagate in the lower ionosphere and thermosphere. The periodic variations between 20 and 35 days are caused mainly by solar flux variations and those between 10 and 20 days can be due to a combination of planetary wave excitation and solar radiation. Dominant periods near 5, 10 and 16-days in the time variations of critical frequency $f_{\mathrm{o}} \mathrm{F} 2$ of the ionospheric F-layer, related to middle atmospheric planetary waves, for three European stations during 1979-1989 have been observed (Lastovicka and Mlch, 1996).

The persistence of ten separate periodic bands, centred about a period of $2,3,5,6.5,9,13.5,18.6$, $21.8,27.4$ and 31.5 days were found in the temporal variations of $f_{\mathrm{o}} \mathrm{F} 2$ at northern middle latitudes during solar cycle 21 (Apostolov and Altadill, 1996) and the long-term variations (seasonal and solar cycle) of their oscillation characteristics were evaluated.

There is overlap between neighbouring bands in separate time intervals and, for longer time scales, the most pronounced quasi-periodic bands are those for 2- 
3, 5-6, 9, 13-14, 18-19 and 28-30 days. These bands may be related with the analogous 2, 5, 10 and 16-day global middle atmosphere oscillations and with the direct solar ionization variations due to the solar rotation (27-29-days) and half solar rotation (13.5-14days) periods. The amplitudes of these $f_{\mathrm{o}} \mathrm{F} 2$ quasiperiodic oscillations are characterized by a considerable variability for different time scales, but with certain regularity in the temporal variations related to seasons and long-term solar and geomagnetic activity variations. The oscillation amplitudes (also in Apostolov et al., 1994, 1995; Altadill, 1996; Altadill et al., 1997) are larger at the equinoxes and lower at the solstices and they are modulated by the long-term geomagnetic variations and simultaneously influenced by the solar activity variations. The maximum probability of occurrence (near $0.9-1)$ of $f_{\mathrm{o}} \mathrm{F} 2$ quasi-2- and 5-day oscillations is in summer half year and minimum (near 0.4) in the winter period (Apostolov et al., 1994, 1995).

Several potential mechanisms have been proposed to explain the influence of the more intensively studied planetary 2-day wave in the middle atmosphere on the arising of upper ionosphere quasi-2-day oscillations. Such mechanisms might contribute to the other planetary wave-type oscillations observed in the upper ionosphere. Pancheva and Lysenko (1988) propose that quasi-2-day oscillations in the electric current system induced by the 2-day wave in the meteor wind region (Ito et al., 1986) may produce such oscillation in the vertical plasma drift, which could generate the same oscillation in the F-region electron concentration. The model investigation by Mikhailov (1983) shows that if the variations of the vertical transport of the neutral constituents $\mathrm{O}$ and $\mathrm{O}_{2}$ cover the entire lower thermosphere, then this leads to in-phase electron concentration variations in the ionospheric regions $\mathrm{E}$ and $\mathrm{F}_{2}$. The development of a quasi-2-day oscillation in the upper ionosphere can be induced by the forcing of such an oscillation in the mesosphere, linked to a particular region of the globe, with further independent development in the F-region (Apostolov et al., 1995). Forbes et al. (1997) assume that the secondary excitation of the quasi-2-day wave due to modulated gravity wave momentum deposition in the E-region or amplitude modulation of upward propagating diurnal and semidiurnal tides can induce such an oscillation of electric fields in the dynamo altitudes.

The solar cycle and seasonal variations of the relative contributions of the main quasi-periodic oscillation bands from 2 to 35 days to the variability of $f_{\mathrm{o}} \mathrm{F} 2$ is the purpose of the present study.

\section{Method of analysis and data}

When estimating the relative influence of arbitrary quasi-periodic oscillation it is usual to compare the amplitude of this oscillation, obtained as a maximum in the amplitude spectrum band, with the amplitude of other band or with the total variation of the investigated time series. The power of influence is underestimated in this way, because the spectral energy is distributed in a series of spectral lines because of the variability of the oscillation period. The integrated content over amplitude spectral area for an arbitrary periodic band is more acceptable as a measure of the power of influence for this band.

The relative contribution of the oscillations in an arbitrary periodic range to the total variations of data series can be quantitatively evaluated using the methodology described by Parish et al., (1994) and based on Parseval's relation. If $x[n], n=-\infty, \infty$, is the discrete time signal and $X(\omega)$ is the spectrum of the signal, the Parseval theorem

$$
\sum_{n=-\infty}^{\infty}|x[n]|^{2}=\frac{1}{2 \pi} \int_{-\pi}^{\pi}|X(\omega)|^{2} \mathrm{~d} \omega
$$

relates the total signal energy (left side of the equation) to its total spectral energy (right side), i.e. how the signal energy is distributed in frequency domain. The energy density spectrum $|X(\omega)|^{2}$ is defined only for finite energy signals.

The quantitative evaluation of the contribution of the variations of an arbitrary periodic range to the total variation of the data series can be expressed as the percentage ratio of the signal energy in this periodic range to the total signal energy. According to Parseval's relation, the percentage ratio of the signal energy is equivalent to the percentage ratio of the spectral energy of this periodic range to the total spectral energy. The total spectral energy can be replaced by total spectral energy for an arbitrary periodic range from $T_{S}$ to $T_{E}$ and we can estimate the percent contributions (PC) for arbitrary periodic bands from $T_{K}$ to $T_{P}$, within the periodic range from $T_{S}$ to $T_{E}$, to the total spectral energy for periodic range from $T_{S}$ to $T_{E}$.

The total spectral energy for the periodic range from $T_{S}$ to $T_{E}$ can be calculated as a sum of squared amplitudes $A(T)$ obtained by a Fourier Transform and the integrated percent contributions for all periods lower than a given value $T_{i}$ can be determined as

$$
P C\left(T_{S} \leq T_{i} \leq T_{E}\right)=\left(\sum_{T=T_{S}}^{T_{i}} A(T)^{2} / \sum_{T=T_{S}}^{T_{E}} A(T)^{2}\right) * 100
$$

The percent contribution of spectral energy for arbitrary periodic band from $T_{K}$ to $T_{P}$ to the total spectral energy for the range from $T_{S}$ to $T_{E}$ is

$$
P C\left(T_{K}, T_{P}\right)=P C\left(T_{S} \leq T_{P} \leq T_{E}\right)-P C\left(T_{S} \leq T_{K} \leq T_{E}\right)
$$

where $T_{S} \leq T_{K}<T_{P} \leq T_{E}$.

For amplitude spectral estimates we use the procedure of correloperiodogram analysis, described in Vitinsky et al., (1986), allowing us to obtain highresolution spectral estimates in the desired periodic range with an arbitrary small step.

In this study we use the $f_{\mathrm{o}} \mathrm{F} 2$ hourly data over Europe from Rome $\left(41.9^{\circ} \mathrm{N}, 12.5^{\circ} \mathrm{E}\right)$, Poitiers $\left(46.5^{\circ} \mathrm{N}, 0.3^{\circ} \mathrm{E}\right)$, 
Kaliningrad $\left(54.7^{\circ} \mathrm{N}, 20.6^{\circ} \mathrm{E}\right)$ and Uppsala $\left(59.8^{\circ} \mathrm{N}\right.$, $\left.17^{\circ} \mathrm{E}\right)$ for the period 17 December 1975-15 January 1987. Each time series contains 97152 values. We chose as starting and ending dates, 17 December and 15 January respectively, because in the present study the moving spectra for intervals 4 or 2 months shifted by 1 month are used and in this way the intervals are centred to date 15 within the month. The few missing values are evaluated by an interpolation between the data of the preceding and following days at the same hour. The four stations lie latitudinally between $42^{\circ} \mathrm{N}$ and $60^{\circ} \mathrm{N}$ and the time interval considered covers the whole solar cycle 21 . This enables the percent contributions of the quasi-periodic oscillations of $f_{\mathrm{o}} \mathrm{F} 2$ to be evaluated as a function of latitude and solar cycle and long-term geomagnetic activities.

\section{Results}

The dominant distribution of power spectral densities for the whole solar cycle by means of averaged periodograms has been obtained in order to illustrate the existence of the main periodic bands of the $f_{\mathrm{O}} \mathrm{F} 2$ quasi-periodic oscillations from 2 to 35 days. The averaged periodogram for each station has been calculated as a mean of 126 periodograms, where each periodogram is obtained for time interval of 2920 hours, equal to four mean-months duration, with a shift of $730 \mathrm{~h}$ (one mean-month duration) between successive time intervals. The periodograms have been calculated in the periodic range from 30 to $960 \mathrm{~h}$, i.e. from 1.25 to 40 days, by a step of $0.2 / 960 \mathrm{~h}^{-1}$ in frequency domain. The size of the four-month interval is three times the 40day period which ensures sufficient statistical stability of the spectral estimates. Figure 1 shows the averaged periodograms for all stations. The relatively minor peaks occur near 2-3 and 19-day periods in all spectra and there are well expressed maxima close to $6,9,14$ and 29 days. The periodic bands labelled here as $2.5,6,9,14$, 19 and 29-day are referred to periodic ranges with limits of preceding and following minima in the periodograms around these magnitude maxima (Table 1).

Note that for the next evaluation of percent contributions of these bands and its temporal variations we have used the interval of $1460 \mathrm{~h}$, equal to two meanmonths duration, shifted by $730 \mathrm{~h}$ (one mean-month duration), to calculate the moving spectra. We chose the interval of two months as an optimal variant between two opposite requirements. The statistical stability of spectral estimates and frequency resolution increase when the time interval increases, but decrease the temporal resolution when the moving spectral analysis is used to evaluate the time variations of spectral characteristics. Moreover, since the quasi-oscillations have a nonstationary character, an increase of interval size may produce an increasing ambiguity in the spectral estimates due to changes of oscillation period with time. The periodograms are calculated for periodic range from Nyquist period of $2 \mathrm{~h}$ to 35 days by a step in frequency domain of $0.2 / 35 \mathrm{day}^{-1}$ (or $0.2 / 840 \mathrm{~h}^{-1}$ ). In this way we can compare the relative contributions of different periodic bands (including daily variations and all bands from 2 to 35 days) to the total variations due to all the periods from $2 \mathrm{~h}$ to 35 -days in the two-month interval.

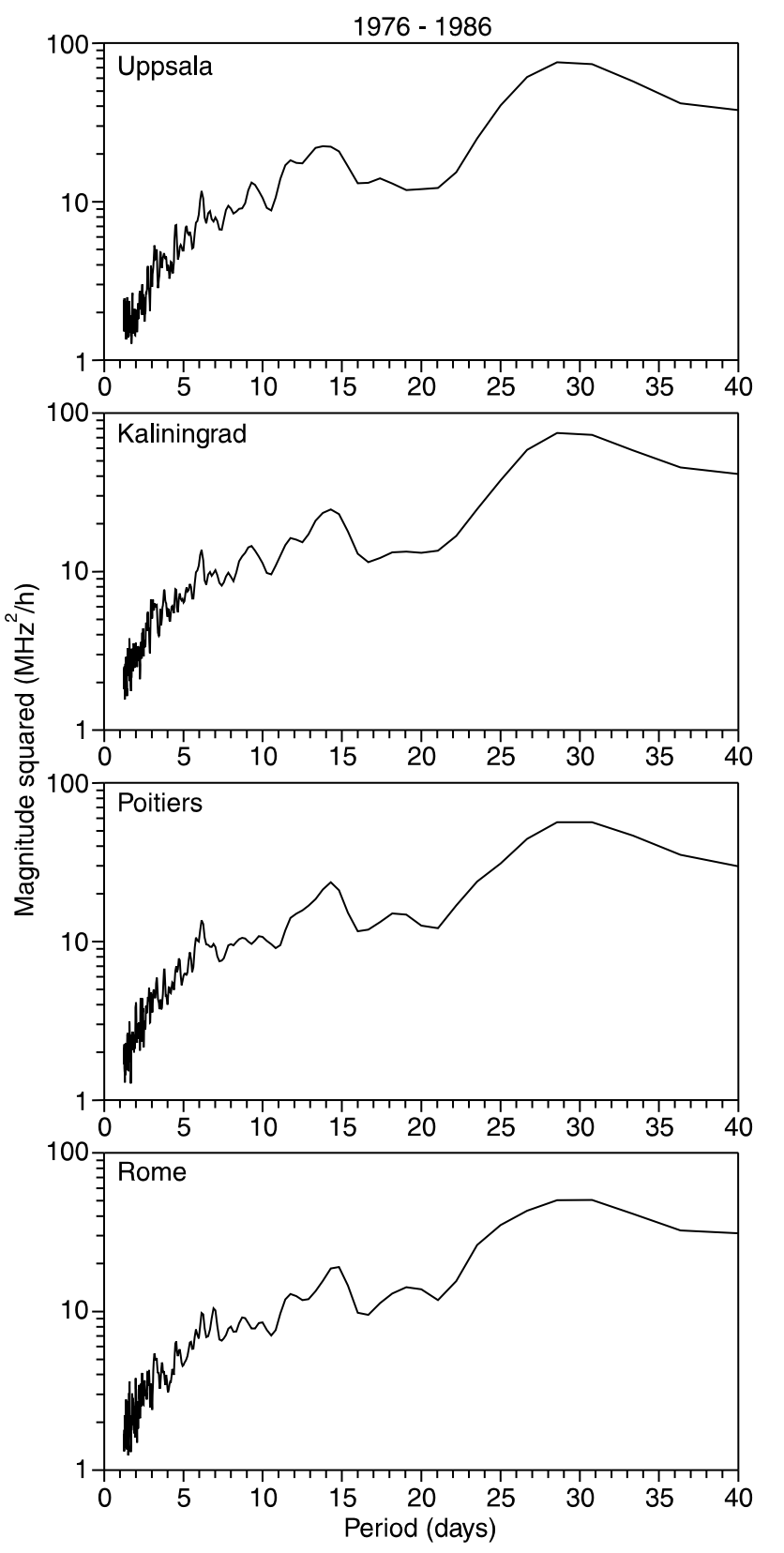

Fig. 1. Averaged periodograms of $f_{\mathrm{o}} \mathrm{F} 2$ in the periodic range from $30 \mathrm{~h}$ to 40 days for the whole solar cycle 21 calculated as a mean of 126 spectra of successive 4-month intervals shifted by 1 month

Table 1. Lower and upper limits of $f_{\mathrm{o}} \mathrm{F} 2$ quasi-periodic bands in the periodic range from 2 to 35 days

\begin{tabular}{lllllll}
\hline $\begin{array}{l}\text { Periodic band } \\
\text { (days) }\end{array}$ & 2.5 & 6 & 9 & 14 & 19 & 29 \\
$\begin{array}{l}\text { Lower limit } \\
\text { days) }\end{array}$ & 1.25 & 4 & 7 & 11 & 16 & 21 \\
$\begin{array}{l}\text { Upper limit } \\
\text { (days) }\end{array}$ & 4 & 7 & 11 & 16 & 21 & 35 \\
\hline
\end{tabular}


For each station, the averaged periodograms for the period 17 December 1975-15 January 1987 is obtained as a mean of 132 periodograms. The averaged periodograms are used to calculate the integrated percent contributions for all periods. The integrated percent contributions for the periods less than a given value between $2 \mathrm{~h}$ to 35 days for the whole solar cycle are presented in Fig. 2. There are no significant differences between the integrated contributions for the four latitudinally separated locations. The integrated percent contribution for all periods from 2 to 35 days is $8 \%$ and the contribution due to the daily variation is $92 \%$. The periodic range of daily variation is taken from 2 to $30 \mathrm{~h}$. The percent contributions of diurnal (78\%) and semidiurnal $(8 \%)$ variations are clearly seen.

The integrated percent contributions are calculated also from the same periodograms separately for two month intervals centred to date 15 for January, March and June, as representative winter, equinoctial and summer months, for years of low (1976) and high (1979) solar activity (Fig. 3). The integrated percent contribution of the periodic range from 2 to 35 days for June depends on the latitude and solar activity level. The contribution increases with latitude for high solar activity from $55 \%$ for Rome to $62 \%$ for Kaliningrad and Uppsala and from 10\% (Rome) to $25 \%$ (Uppsala) for low solar activity. The contribution for high solar activity for March varies from 8\% (Rome) to $15 \%$ (Uppsala) and for low activity from 5\% to $10 \%$ respectively. There are no clearly expressed latitudinal and solar cycle dependencies for January, the mean contribution for periods from 2 to 35 days is near $2 \%-5 \%$.
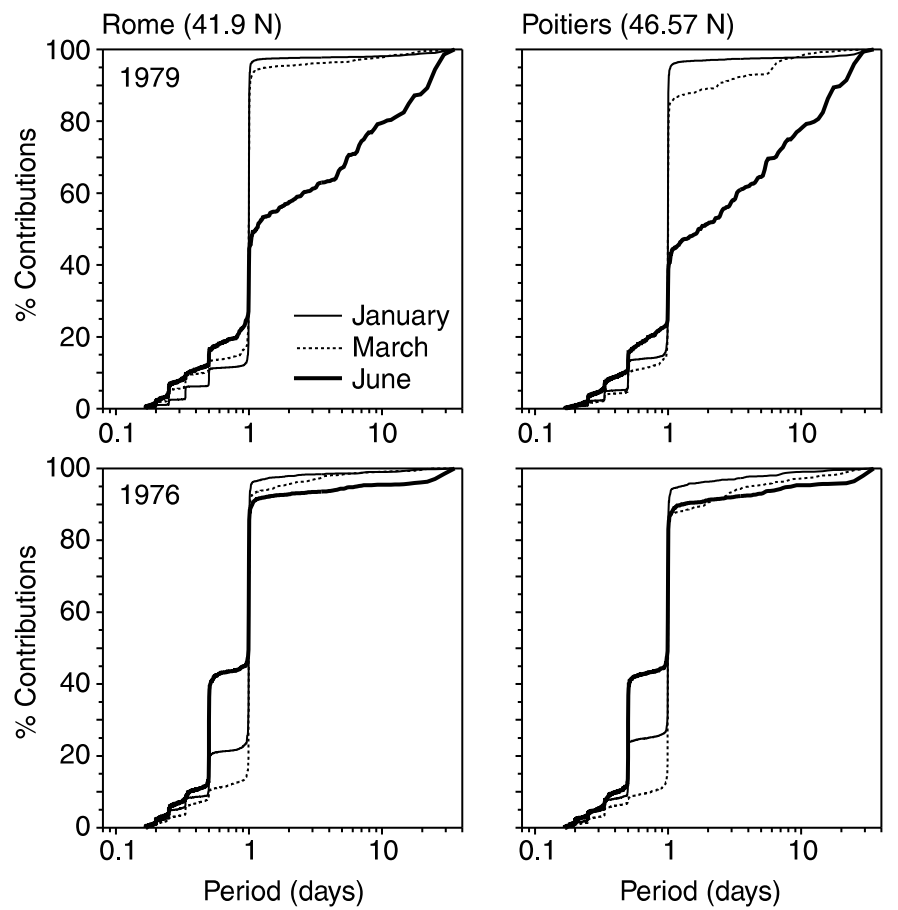

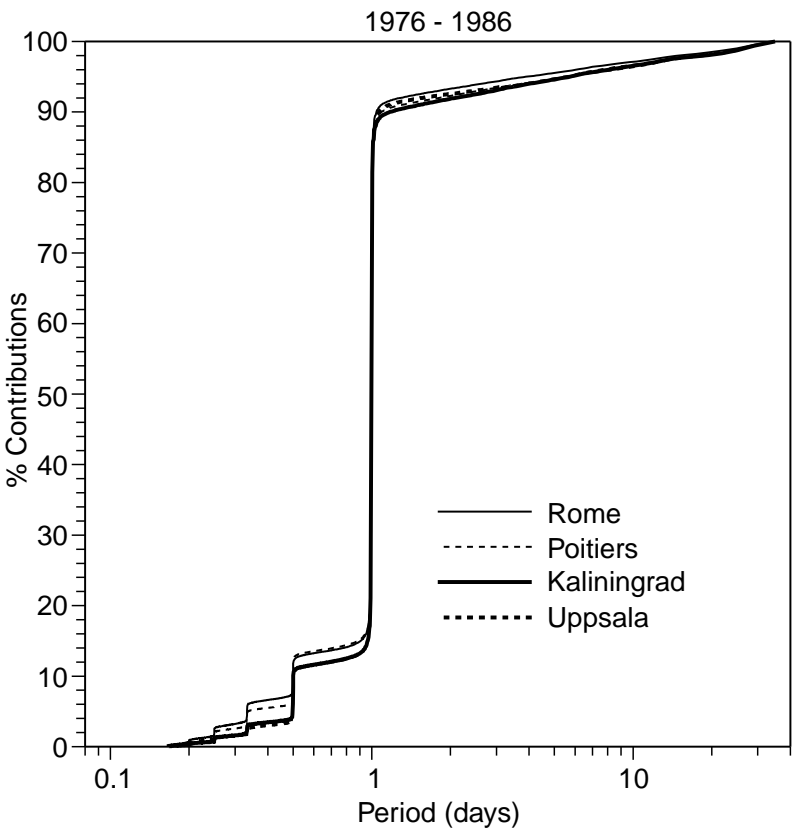

Fig. 2. Integrated percent contributions for periods lower than a given value in the periodic range from $4 \mathrm{~h}$ to 35 days for the whole solar cycle

The temporal and latitudinal variations of the integrated percent contributions of each periodic band have been calculated from the periodograms for each month and station. The results are given in Fig. 4. The long-term variations of sunspot numbers $\mathrm{Rz}$ (thick line) and of geomagnetic activity index Ap (dashed line) are presented at the same figure in the top panel. They have
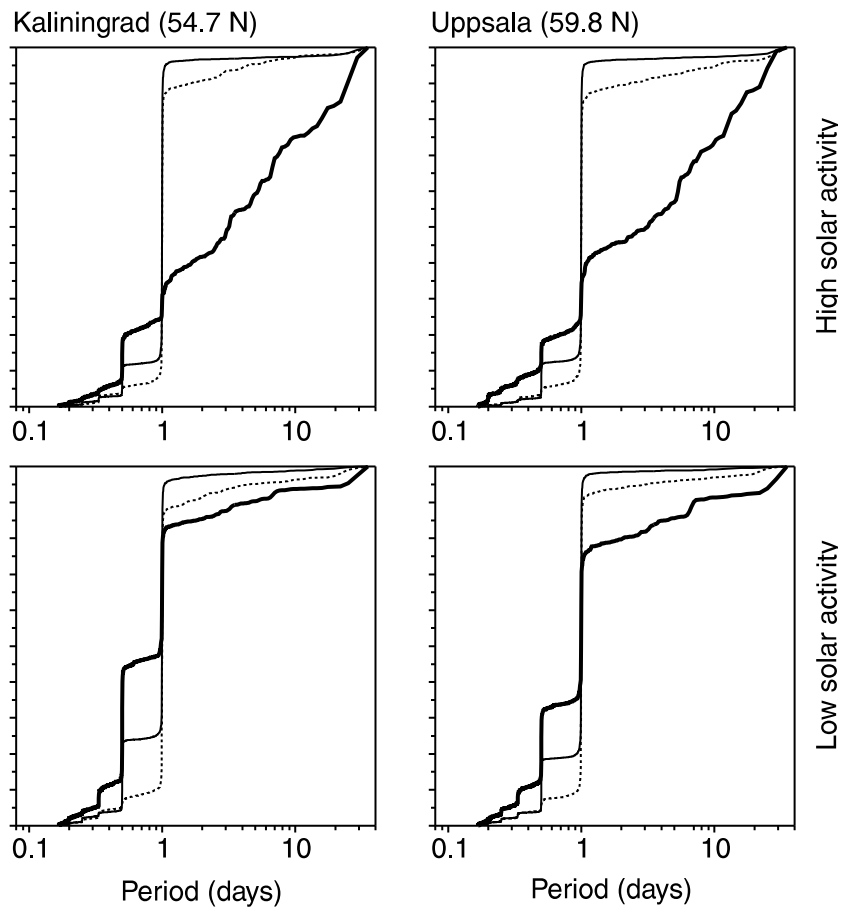

Fig. 3. Integrated percent contributions for periods lower than a given value in the periodic range from $2 \mathrm{~h}$ to 35 days over 2 months intervals centred for January, March and June for high solar activity (1979 - upper panel) and for low solar activity (1976 - lower panel) 


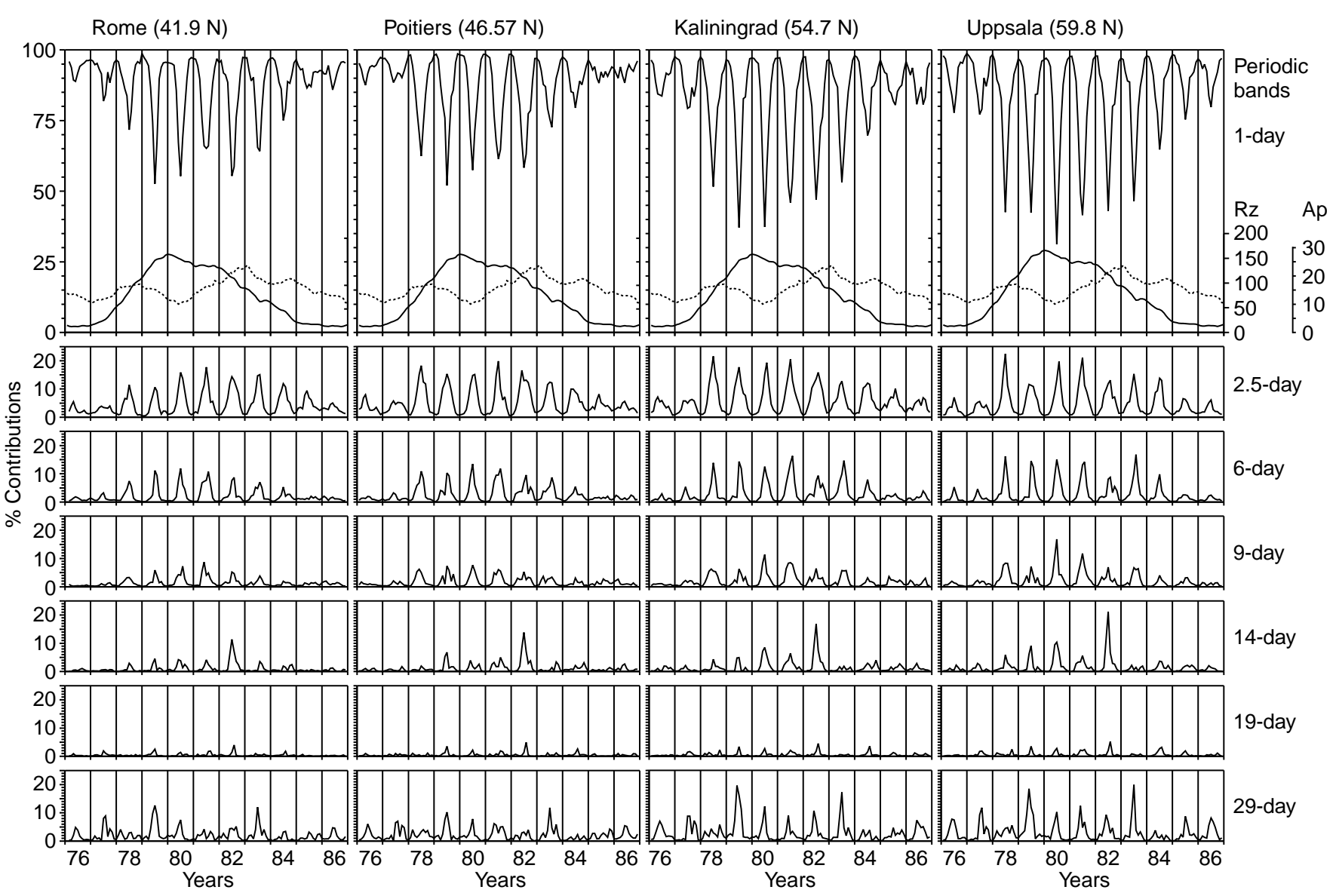

Fig. 4. Annual and solar cycle variations of the integrated percent contributions for daily variation and for 2.5, 6, 9, 14, 19 and 29-day periodic bands. The 12-month smoothed averages of monthly means

been obtained by smoothing the annual variations with the 12-month running means with half weights of the first and last months, i.e. by the well-known procedure for obtaining the R12.

The observations suggest that there is a clearly expressed seasonal variation of percent contributions with maximum at the summer solstice and minimum at the winter one for all periodic bands of the quasiperiodic oscillations and vice versa for the percent contributions of the daily variation. The percent contributions for summer half year for all quasi-periodic bands increases when the latitude increases. The decrease of percent contribution of daily variation for summer when the latitude increases is clearly seen also.

There is a solar cycle and long-term geomagnetic activity modulation of percent contribution variations, and this is especially well developed for the summer values. The enhanced percent contributions for 2.5 - and 6-day periodic bands are for 1978 and 1982-1983, in the increasing and decreasing parts of the solar cycle, when the first and second geomagnetic activity maxima exist. For the 9-day periodic band there is an enhancement of percent contributions in the years around solar activity maximum (1978-1983) and for 14-day band the higher value is in 1982. The percent contributions for the 19day band are practically negligible during whole solar of sunspot numbers Rz (thick line) and geomagnetic activity index Ap (dashed line) are presented in the upper panel

cycle, and for 29-day band they show a nearly uniform distribution except for the enhancements during 1979 and 1983.

The previous investigations (Apostolov and Altadill, 1996) concerning the existence and persistence of $f_{\mathrm{o}} \mathrm{F} 2$ quasi-periodic oscillations with periods from 2 to 35 days, as well as in the equatorial electrojet variations (Parish et al., 1994), suggest that the oscillations, referred to here as 2.5-, 6- and 9-day periodic bands, may be interpreted in terms of middle atmosphere planetary wave influence. The oscillations of 14- and 29day periodic bands are related to solar flux variations. The origin of the 19-day periodic band is quite controversial. Forbes and Leveroni (1992) reported a quasi-16day oscillation in the E- and F-regions of the equatorial ionosphere, connected to upward penetration of a free Rossby mode excited in the winter stratosphere. Parish et al. (1994) found quasi-periodic variations near 16-day with characteristics of planetary waves. The investigation by Altadill (1996) shows that the dominant period of this oscillation between 1970-1990 is near 18-day for lower, middle and upper ionosphere and suggests a possible solar influence on this oscillation.

We separate the relative contributions of the main sources for the $f_{\mathrm{o}} \mathrm{F} 2$ variability, caused by quasi-periodic oscillations from 2 to 35 days, by summarizing the 
percent contributions for 2.5-, 6- and 9-day bands for planetary wave activity and 14- and 29-day bands for solar flux variations. The percent contributions for the 19-day band have not been considered because they are negligible. The sums of percent contributions for periodic bands of 2.5-, 6- and 9-day (2.5-9 day) and of 14and 29-day (14-29 day) are calculated from the data, presented in Fig. 4. The results are displayed in Fig. 5 and provide a comparative evaluation of the contributions of quasi-periodic oscillations due to planetary wave activity (middle panel), solar flux variations (lower panel) and the contribution of the relatively stable daily variation (upper panel).

The percent contributions for the three periodic bands of daily variation, planetary wave activity and solar flux variations, for the two extrema of annual variation (winter and summer solstices) for each of the four stations are given in Table 2. The percent contributions for winter solstice are averaged for the whole period 1976-1986, because there are very weak solar cycle variations for each of the periodic bands. The contributions for summer solstice are averaged for the years of low $(\mathrm{Rz}<50)$, mean $(50<\mathrm{Rz}<100)$ and high $(\mathrm{Rz}>100)$ solar activity. From the results presented in Fig. 5 and Table 2 the next conclusions can be made.

The increase of the contributions of the daily variation for winter solstice and the decrease for summer one when the latitude increases are in accordance with the well-known equivalent $f_{\mathrm{o}} \mathrm{F} 2$ diurnal and semi-diurnal amplitude variations with latitude. The limits of decrease with latitude for the summer solstice decreases when the solar activity increases. This decrease for low solar activity varies from $87.5 \%$ (Rome) to $77.5 \%$ (Uppsala) and for high solar activity varies from $57.1 \%$ (Rome) to $39.5 \%$ (Uppsala).

The percent contributions of the quasi-periodic oscillations bands of planetary wave activity and solar flux variations for winter solstice are negligible, between $0.7 \%$ and $3.6 \%$. Nevertheless, the contributions of planetary wave activity are 2-4 times higher than that of solar flux variations. The energy contributions for these two bands during the summer solstice have a clear tendency to increase when latitude increases. The percent contributions of planetary waves for low solar activity have a small superiority over those of solar flux variations, they are 1.5-2 times higher for high solar activity and 2.5-3 times higher for mean solar activity. The latter is due to the fact that during the years of mean solar activity are the two maxima of long-term geomagnetic activity and that the modulation effect of geomagnetic activity variations on the 2.5- and 6-day bands of planetary wave activity is significant (see Fig. 4 and commentary).

The energy contributions for all quasi-periodic oscillations from 2 to 35 days for summer solstice are most significant for the years of high solar activity. The integrated energy contribution for periods from 2 to 35 days averaged for the years of high solar activity (19791982 ) is from $42.7 \%$ (Rome) to $59.2 \%$ (Uppsala).

\section{Summary and conclusions}

The energy contributions of different periodic bands of quasi-periodic oscillations from 2 to 35 days to the
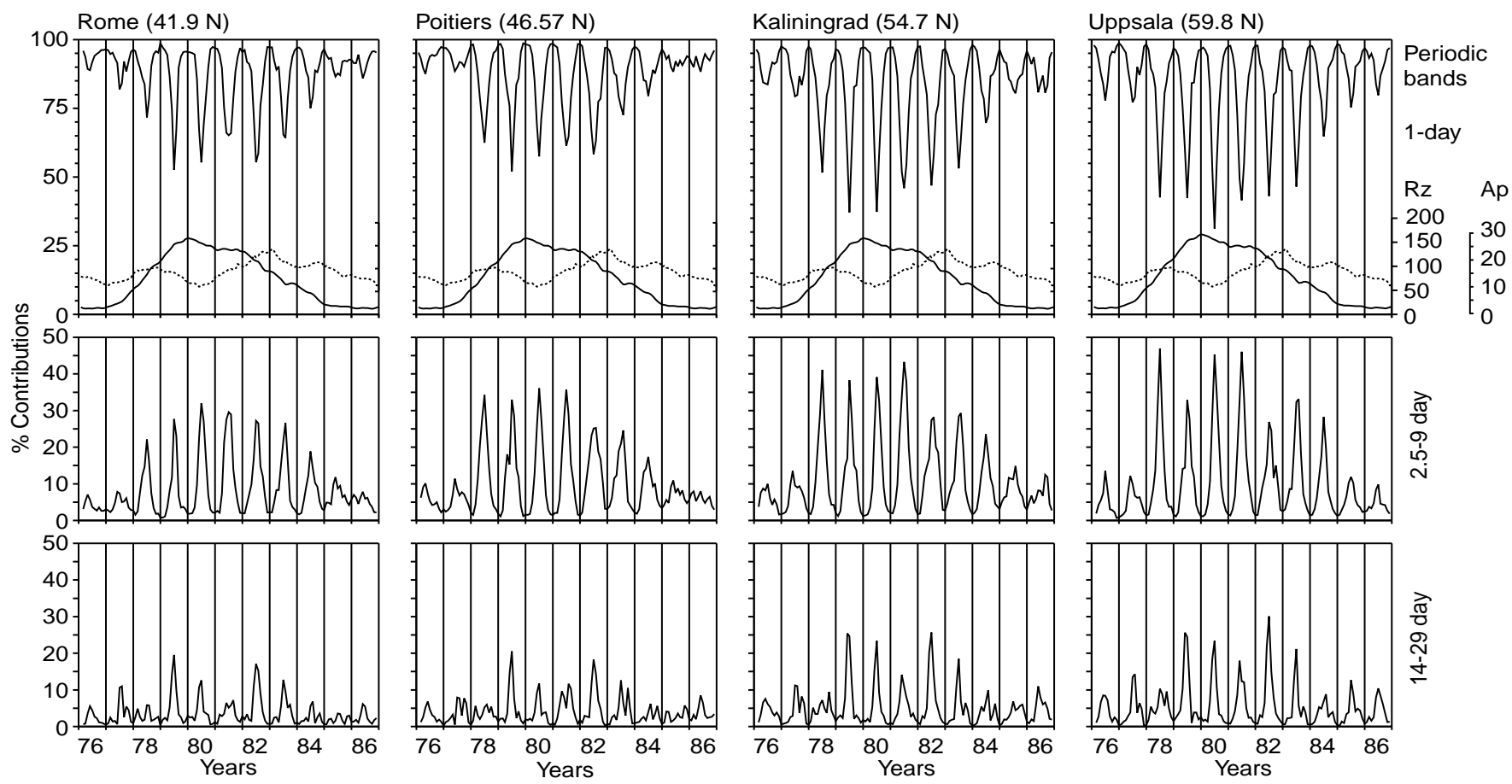

Fig. 5. Annual and solar cycle variations of the integrated percent contributions for daily variation, for periodic bands due to planetary wave activity (2.5-9 day) and solar flux variations (14-29 day) 
Table 2. Relative contributions of the main sources for $f_{\mathrm{OF}} 2$ variability in relation to solar activity, season and latitude. Row (1) is for contributions of daily variations, (2) for contributions linked with the planetary wave activity (all periodic bands from 2.5- to 9-day), and (3) for contributions associated with solar flux variations (14- and 29-day)

\begin{tabular}{|c|c|c|c|c|c|c|}
\hline & & Rome $41.9 \mathrm{~N}$ & Poitiers $46.5 \mathrm{~N}$ & Kaliningrad $54.7 \mathrm{~N}$ & Uppsala $59.8 \mathrm{~N}$ & \\
\hline \multirow{2}{*}{\multicolumn{2}{|c|}{ Winter }} & 95.2 & 96.2 & 96.7 & 97.8 & (1) \\
\hline & & 3.6 & 3.1 & 2.4 & 1.6 & (2) \\
\hline \multicolumn{2}{|l|}{ Solstice } & 1.2 & 0.7 & 0.9 & 0.6 & (3) \\
\hline \multirow{6}{*}{ Summer } & & 87.5 & 87 & 82.2 & 77.5 & (1) \\
\hline & $\mathrm{Rz}<50$ & 6.4 & 6.7 & 9.7 & 11.3 & (2) \\
\hline & & 6 & 6.3 & 8 & 11.2 & (3) \\
\hline & & 71.1 & 68.7 & 58.2 & 51.2 & (1) \\
\hline & $50<\mathrm{Rz}<100$ & 20.9 & 24.1 & 31 & 35.8 & (2) \\
\hline & & 8 & 7.2 & 10.8 & 12.7 & (3) \\
\hline \multirow[t]{3}{*}{ Solstice } & & 57.1 & 53.5 & 41.8 & 39.5 & (1) \\
\hline & $\mathrm{Rz}>100$ & 29.1 & 32.5 & 37 & 37.7 & (2) \\
\hline & & 13.6 & 13.9 & 20.1 & 21.5 & (3) \\
\hline
\end{tabular}

variability of $f_{\mathrm{o}} \mathrm{F} 2$ are found in the present study. Solar cycle and long-term geomagnetic variations as well as annual variations of the contributions for the four latitudinally separated locations from $42^{\circ} \mathrm{N}$ to $60^{\circ} \mathrm{N}$ are evaluated. The integrated percent contribution for periodic bands from 2.5- to 9-days of planetary wave activity, averaged for the whole solar cycle, is near 5\% and for 14- and 29-day bands of solar flux variations is near 3\% (Fig. 2). The remaining $92 \%$ are due to the daily variation. An averaging over the whole solar cycle would bring us to the incorrect conclusion that the energy contribution of quasi-periodic oscillations from 2 to 35 days is negligible. Nevertheless, the annual and solar cycle variations of the percent contributions (Figs. 4, 5 and Table 2) show an important energy contribution by these quasi-periodic oscillations to the $f_{\mathrm{o}} \mathrm{F} 2$ variability:

1. A clear annual variation of percent contributions for all quasi-periodic bands from 2 to 35 days with minimum at the winter solstice and maximum at the summer is observed and vice versa for contributions of daily variations;

2 . The contributions are modulated by the solar cycle and simultaneously influenced by the long-term geomagnetic activity variations.

3. The energy contributions for the quasi-periodic bands from 2 to 35 days increase when the latitude increases, and the reverse happens for the contributions of daily variations.

4. The main result obtained here suggests that the energy contributions of the planetary wave-type oscillations dominate over those of the solar flux variations for the whole investigated period (1976-1986) and latitude range $\left(42^{\circ} \mathrm{N}-60^{\circ} \mathrm{N}\right)$. The $f_{\mathrm{o}} \mathrm{F} 2$ energy due to the planetary wave activity can reach up to $45 \%$ (for summer solstice of 1980 , Uppsala) of the total energy in the periodic range from $2 \mathrm{~h}$ to 35 days.

The present investigation is not able to specify the importance of the proposed mechanisms, briefly summarized in the Introduction, concerned with beginning and development of the quasi-periodic oscillations in the upper ionosphere due to planetary wave activity in the middle neutral atmosphere.
A follow-up project to this investigation will be the use of a global data base and to move in the direction of predictive ionospheric capability.

Acknowledgements. E. Apostolov expresses his thanks to the France Telecom/CNET for supporting him in this work over three month's stay at Centre Lannion B, France. D. Altadill would like to acknowledge the Commission for Universities and Research of Catalonia Government (CIRIT-grant 1996BEAI200002) and France Telecom/CNET for supporting him for two month's stay at Lannion, France.

Topical Editor D. Alcaydè thanks Lj. R. Cander and H. Parish for their help in evaluating this paper.

\section{References}

Altadill, D., On the 18-day quasi-periodic oscillations in the ionosphere, Ann. Geophysicae, 14, 716-724, 1996.

Altadill, D., E. M. Apostolov, and L. Alberca., Some seasonal hemispheric similarities in $f_{\mathrm{o}} \mathrm{F} 2$ quasi-2-day oscillations, J. Geophys. Res., 102, 9737-9739, 1997.

Apostolov, E. M., and D. Altadill, Ten periodic bands of $f_{\mathrm{o}} \mathrm{F} 2$ quasiperiodic oscillations from 2 to 35 days, Bulg. Geophys. J., 22(3), 20-24, 1996.

Apostolov, E. M., L. Alberca, and D. Altadill, Solar cycle and seasonal behaviour of quasi-two and five-day oscillations in the time variations of $f_{\mathrm{o}} \mathrm{F} 2$, Ann. Geofisica, 37(2), 187-192, 1994.

Apostolov, E. M., D. Altadill, and L. Alberca, Characteristics of quasi-2-day oscillations in the $f_{\mathrm{o}} \mathrm{F} 2$ at northern middle latitudes, J. Geophys. Res., 100, 12163-12171, 1995.

Chen, P. R., Two-day oscillation of the equatorial ionization anomaly, J. Geophys. Res., 97, 6343-6357, 1992.

Forbes, J. M., and S. Leveroni, Quasi 16-day oscillation in the ionosphere, Geophys. Res. Lett., 19, 981-984, 1992.

Forbes, J. M., R. Guffee, X. Zhang, D. Fritts, D. Riggin, A. Manson, C. Meek, and R. A. Vincent, Quasi 2-day oscillation of the ionosphere during summer 1992, J. Geophys. Res., 102, 7301-7305, 1997.

Fraser, G. J., The 5-day wave and ionospheric absorption, J. Atmos. Terr. Phys., 39, 121-124, 1977.

Ito, R., S. Kato, and T. Tsuda, Consideration of an ionospheric wind dynamo driven by a planetary wave with a two-day period, J. Atmos. Terr. Phys., 48, 1-13, 1986.

Lastovicka, J., and P. Mlch, Solar cycle effect on oscillations in the period range of 2-20 days in the $F$ region of the ionosphere, Ann. Geofisica, 39(4), 783-790, 1996.

Mikhailov, A. V., Possible mechanism for in-phase variation of electron concentration in the ionospheric regions $E$ and $F 2$ (in Russian), Geomagn. Aeron., 23, 557-561, 1983. 
Pancheva, D., Travelling quasi-two-day fluctuations in the summer F-region, C. R. Acad. Bulg. Sci., 41(11), 41-44, 1988.

Pancheva, D., and I. Lysenko, Quasi-two-day fluctuations observed in the summer $\mathrm{F}$ region electron maximum, Bulg. Geophys. J., 14(2), 41-51, 1988.

Pancheva, D., L. Alberca, and B. de la Morena, Simultaneous observation of the quasi-two-day variations in the lower and upper ionosphere over Europe, J. Atmos. Terr. Phys., 56, 43-50, 1994.
Parish, H. F., J. M. Forbes, and F. Kamalabadi, Planetary wave and solar emission signatures in the equatorial electrojet, J. Geophys. Res., 99, 355-368, 1994.

Salby, M. L., Survey of planetary scale travelling waves: the state of theory and observations, Rev. Geophys. Space Phys., 22, 209236, 1984.

Vitinsky, Y. I., M. Kopecky, and G. V. Kuklin, Statistics of sunspotcreating activity (in Russian), Nauka, Moscow, 1986. 